\title{
Intestinal Barrier Biomarker ZO1 and Endotoxin Are Increased in Blood of Patients With COVID-19-associated Pneumonia
}

\author{
STELIOS F. ASSIMAKOPOULOS ${ }^{1}$, STYLIANOS MASTRONIKOLIS ${ }^{2}$, ANNE-LISE DE LASTIC ${ }^{3}$, \\ DIAMANTO ARETHA ${ }^{4}$, DIMITRIS PAPAGEORGIOU ${ }^{1}$, THEODORA CHALKIDI $^{1}$, IOANNA OIKONOMOU ${ }^{1}$, \\ CHRISTOS TRIANTOS ${ }^{1}$, ATHANASIA MOUZAKI $^{3}$ and MARKOS MARANGOS ${ }^{1}$ \\ ${ }^{1}$ Department of Internal Medicine, University of Patras Medical School, Patras, Greece; \\ ${ }^{2}$ School of Medicine, University of Crete, Heraklion, Greece; \\ ${ }^{3}$ Division of Hematology, Department of Internal Medicine, University of Patras Medical School, Patras, Greece; \\ ${ }^{4}$ Department of Anesthesiology and Intensive Care Medicine, University of Patras Medical School, Patras, Greece
}

\begin{abstract}
Background/Aim: The present study was undertaken to investigate (i) whether hospitalized patients with COVID-19 pneumonia present intestinal barrier dysfunction with consequent translocation of endotoxin into the systemic circulation and (ii) whether intestinal barrier biomarkers have any prognostic role in terms of progression to severe respiratory failure. Patients and Methods: In this prospective study, 22 patients with COVID-19-associated pneumonia and 19 patients with non-COVID-19-related community-acquired pneumonia (CAP group) were studied while 12 healthy persons comprised the control group. Blood samples were collected on admission and analysed for serum levels of endotoxin and zonula occludens-1 (ZO1). Clinical courses regarding progression to severe respiratory failure $(S R F)$ requiring mechanical ventilation were recorded. Results: Patients with COVID-19-associated pneumonia and patients with CAP presented significantly higher serum endotoxin and ZOI concentrations on admission as compared to healthy controls. There was no difference in endotoxin levels between patients with COVID-19-related pneumonia and patients with CAP. In patients with COVID19-related pneumonia, serum endotoxin concentrations were positively correlated with $C$-reactive protein and ferritin values. There were no significant differences in serum
\end{abstract}

This article is freely accessible online.

Correspondence to: Stelios F. Assimakopoulos, MD, Ph.D., Assistant Professor, Department of Internal Medicine and Division of Infectious Diseases, University of Patras Medical School, Patras 26504, Greece. Tel: +30 2610999583, Fax: +30 2610993982, e-mail: sassim@upatras.gr

Key Words: COVID-19, pneumonia, gut barrier, bacterial translocation, endotoxin, ZO1 endotoxin and ZO1 concentrations between patients with severe and not severe COVID-19-related pneumonia, nor between patients who developed SRF and those who did not Conclusion: Patients with COVID-19-related pneumonia present intestinal barrier dysfunction leading to systemic endotoxemia. Admission values of endotoxin and ZO1 do not have any prognostic role for progression to $S R F$.

Although the vast majority of patients infected with SARSCoV-2 will suffer from a mild respiratory illness, some patients develop severe complications, such as acute respiratory distress syndrome, renal and hepatic dysfunction, coagulopathy with thromboses and acute cardiac injury, increasing the risk of mortality (1). In these severely ill patients with multiple organ dysfunction syndrome, usually no infectious bacterial focus is identified and blood cultures are negative but the clinical picture is compatible with sepsis, supporting the concept of viral sepsis (2).

The intestine plays a central role in the pathophysiological sequence of events that lead from sepsis to multiple organ dysfunction, being characterized as the 'motor' of sepsis (3). Enterocytes in the small intestine and colon express the angiotensin-converting enzyme 2 receptor and can therefore be infected by the SARS-CoV-2 virus (4). A multicenter study with 204 patients with COVID-19 from Hubei, China, showed that half of the patients experienced gastrointestinal symptoms and one-third had diarrhoea. In addition, SARSCoV-2 RNA is commonly detected in faeces from patients with COVID-19, with a 3 -fold increased risk of detection in those with diarrhoea (5). Previous studies have provided evidence for intestinal microbiota alterations, while endotoxemia and circulating bacteriome have been detected in critically ill patients with COVID-19 $(6,7)$. Bacterial and endotoxin translocation might activate the release of cytokines and other proinflammatory mediators, producing structural and functional deleterious effects on remote 
in vivo 35 : $2483-2488(2021)$

Table I. Baseline characteristics in patients with non-COVID-19-related community-acquired pneumonia (CAP) and COVID-19-related pneumonia and progression to severe respiratory failure $(S R F)$.

\begin{tabular}{|c|c|c|c|c|}
\hline \multicolumn{2}{|l|}{ Characteristic } & \multirow{2}{*}{$\frac{\text { CAP }(n=19)}{9 / 10}$} & \multirow{2}{*}{$\begin{array}{c}\text { COVID-19-related pneumonia }(\mathrm{n}=22) \\
11 / 11\end{array}$} & \multirow{2}{*}{$\frac{p \text {-Value }}{0.86}$} \\
\hline Gender, n & Male/female & & & \\
\hline Age, years & Median (IQR) & $60(55-66)$ & $64(55-69)$ & 0.28 \\
\hline $\mathrm{CRP}, \mathrm{mg} / \mathrm{dl}$ & Median (IQR) & $9(3-15)$ & $5(2-13)$ & 0.18 \\
\hline D-Dimers, $\mu \mathrm{g} / \mathrm{ml}$ & Median (IQR) & $0.9(0.4-2.9)$ & $1.7(0.8-3)$ & 0.17 \\
\hline Ferritin, ng/dl & Median (IQR) & $345(195-928)$ & $328(181-1574)$ & 0.77 \\
\hline Fibrinogen, mg/dl & Mean \pm SD & $561 \pm 200$ & $619 \pm 108$ & 0.20 \\
\hline Severe pneumonia, $\mathrm{n}$ & Yes & $8 / 19$ & $9 / 22$ & 0.93 \\
\hline Positive blood culture, $\mathrm{n}$ & Yes & $0 / 19$ & $0 / 22$ & - \\
\hline Progression to SRF, $\mathrm{n}$ & Yes & $4 / 19$ & $7 / 22$ & 0.43 \\
\hline
\end{tabular}

CRP: C-Reactive protein; SD: standard deviation.

organs, thus promoting the development of viral sepsis and multiple organ dysfunction syndrome.

The present study was undertaken to investigate (i) whether hospitalized patients with COVID-19-related pneumonia present intestinal epithelial barrier dysfunction with consequent translocation of endotoxin into the systemic circulation and (ii) whether intestinal barrier biomarkers play any prognostic role in terms of progression to severe respiratory failure (SRF).

\section{Patients and Methods}

Patients. We prospectively studied 22 consecutive patients hospitalized at the University Hospital of Patras from March 3 to May 3, 2020, diagnosed with COVID-19-related pneumonia according to established criteria, requiring confirmation of SARSCoV-2 infection by positive real-time reverse transcriptionpolymerase chain reaction assay of a nasopharyngeal swab sample (8). The exclusion criteria were as follows: Malignancy, rheumatic diseases, renal diseases, gastrointestinal diseases (e.g. celiac disease, inflammatory bowel disease, gastrointestinal bleeding or intestinal surgery in the previous 4 weeks), infections during the previous 4 weeks, alcohol abuse during the previous 4 weeks, elevated serum or urine amylase and treatment during the previous month with medications that might act on tight junction (TJ) regulation such as antibiotics, corticosteroids, nonsteroidal anti-inflammatory drugs and antioxidants (vitamins $\mathrm{C}$ and $\mathrm{E}$, allopurinol, $N$-acetyl-cysteine) (9). Nineteen age- and sex-matched patients with non-COVID-19 (negative molecular testing of respiratory secretions for SARS-CoV2) community-acquired pneumonia (CAP) of the same severity and 12 healthy persons were also included for comparisons. CAP was diagnosed for patients who were not hospitalized for the previous 90 days and presented with all the following: (a) At least two out of four clinical signs compatible with a lower respiratory tract infection i.e., dyspnoea, purulent expectoration, cough and auscultatory rales; and (b) new infiltrate on chest X-ray. Blood was sampled for analyses of investigated parameters within $24 \mathrm{~h}$ of admission. Classification of pneumonia severity was based on the American Thoracic Society guidelines (10). Patients with COVID-19-related pneumonia were treated according to the National Institutes of Health and the National (Greek) Public Health Organization treatment guidelines (8). They were followed-up for 14 days for development of severe respiratory failure $(\mathrm{SRF})$ (defined as $\mathrm{PO}_{2} / \mathrm{FiO}_{2}<200$ ) requiring invasive or non-invasive mechanical ventilation. Patients with SRF underwent computed tomographic pulmonary angiography for exclusion of thromboembolic disease. Patients were enrolled in the context of an infectious diseases and sepsis protocol approved by the Regional Research Ethics Committee (9632/17-05-2016). Our study was carried out in accordance with the ethical guidelines of the 2003 Declaration of Helsinki.

Serum endotoxin and zonula occludens-1 (ZO1) measurements. The concentrations of endotoxin and ZO1 in the serum of patients with COVID-19-related pneumonia or CAP and healthy controls were measured by enzyme-linked immunosorbent assay using commercially available kits Endotoxin (cat\#abx051541; Abbexa Ltd, Cambridge Science Park, Cambridge, UK) (range=0.015-2 EU/ml, sensitivity<0.005), ZO1 (TJP1; cat\#EH15434; Finetest, Wuhan Fine BiotechCo, Wuhan, PR China) (range=0.156-10 ng/ml, sensitivity $=0.094 \mathrm{ng} / \mathrm{ml}$ ) as per the manufacturer's instructions.

Statistical analyses. Data were analysed using the SPSS statistical package for Windows (version 25.0; IBM, Armonk, NY, USA) and GraphPad Prism (version 9.1.0, GraphPad Software Inc., San Diego, CA, USA). Normality of data was tested using the Shapiro-Wilk test. All parameters exhibited a non-normal distribution except fibrinogen. Comparisons were performed using the nonparametric analysis of variance (Kruskal-Wallis test) followed by a post-hoc Mann-Whitney $U$-test (non-normally distributed data) or with one-way analysis of variance followed by post-hoc Student's $t$-test (normally distributed data). Subanalyses of patients with COVID-19-related pneumonia (severe/non-severe, progressors to SRF or not) were performed with the Mann-Whitney $U$-test. Results are expressed as the median (interquartile range) for non-normally distributed data or the mean \pm standard deviation for normally distributed data. The chisquared test, with Yates' correction if required, was used to compare the proportional data. Correlations were estimated by a nonparametric Spearman correlation test. All tests were two-tailed and a $p$-value of less than 0.05 was considered significant.

\section{Results}

Patient characteristics. The baseline characteristics of the patients with COVID-19-related pneumonia and those with 


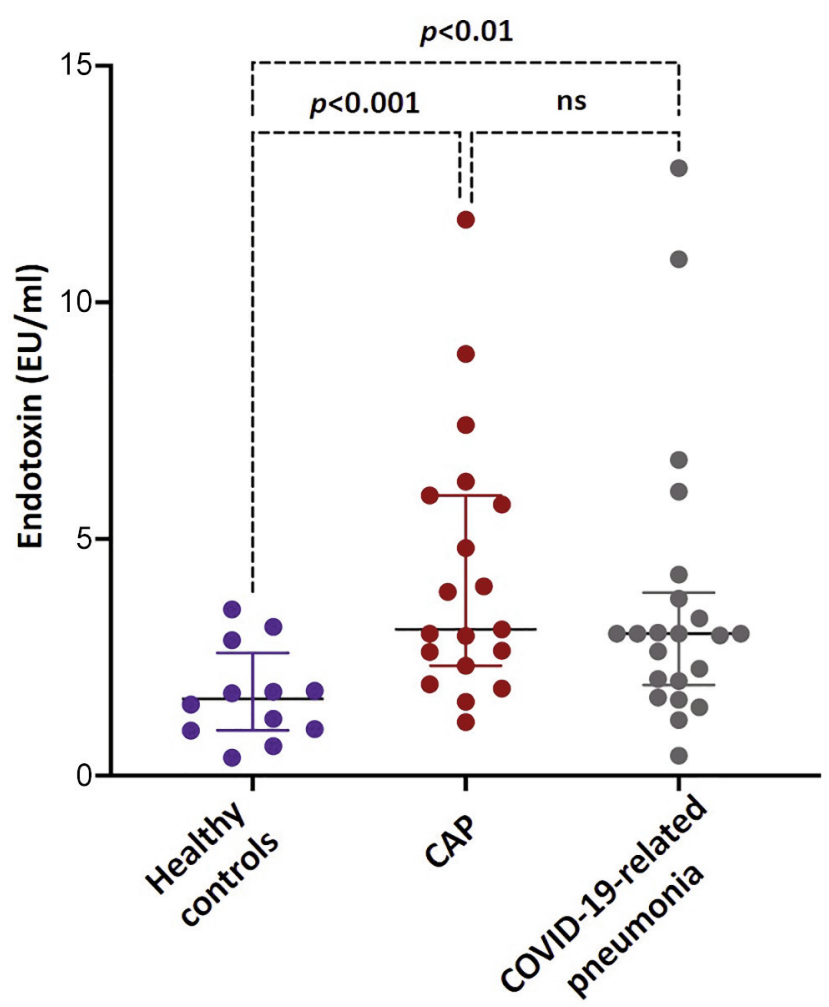

Figure 1. Serum endotoxin levels in healthy controls, patients with nonCOVID-19-related community-acquired pneumonia (CAP) and those with COVID-19-related pneumonia. Lines and bars represent median and interquartile range, respectively ns: Non-significant.

CAP, including age, sex, CRP, ferritin, D-dimers, fibrinogen, as well as progression to SRF are shown in Table I. There were no statistically significant differences in age, gender, CRP, D-dimers, fibrinogen and ferritin levels between patients who presented CAP and those with COVID-19related pneumonia. All blood cultures that were taken on admission were negative. Progression to SRF was observed in $7 / 22$ patients with COVID-19-related pneumonia and in $4 / 19$ with CAP $(p>0.05)$.

Endotoxin. Patients with COVID-19-related pneumonia and those with CAP presented significantly higher serum endotoxin concentrations as compared to healthy controls $(p<0.01$ and $p<0.001$, respectively) (Figure 1). There was no statistically significant difference in endotoxin levels between patients with COVID-19-related pneumonia and those with CAP. In addition, there were no statistically significant differences in serum endotoxin concentrations between patients with severe and those with not severe COVID-19-related pneumonia (Figure 2A upper panel), nor between patients who progressed to SRF and those who did not (Figure 2A lower panel).
ZO1. Patients with COVID-19-related pneumonia and patients with non-COVID-19 CAP presented significantly higher levels of serum $\mathrm{ZO} 1$ compared to healthy controls $(p<0.001$, respectively) (Figure 3$)$. There was no statistically significant difference in $\mathrm{ZO} 1$ levels between patients with COVID-19-related pneumonia and those with CAP. There were no statistically significant differences in the levels of ZO1 between patients with severe and those with not severe COVID-19-related pneumonia (Figure 2B upper panel) nor between patients who progressed to SRF and those who did not (Figure 2B lower panel).

Correlations. In patients with COVID-19-related pneumonia, serum endotoxin concentrations were positively correlated with CRP $(\mathrm{r}=0.52, p<0.05)$ and ferritin values $(\mathrm{r}=0.54$, $p<0.05$ ) (Figure 4).

\section{Discussion}

In this COVID-19-related pneumonia cohort with 22 patients, we have shown that there was significant systemic release of endotoxin, comparable to that in non-COVIDrelated CAP. Previous studies have shown that CAP induces significant endotoxemia, which is of greater magnitude than that observed in other infections such as urinary, intraabdominal and ventilator-associated pneumonia (11). Considering that the bacterial aetiology of CAP implicates mainly Gram-positive pathogens, while endotoxin is a compound of the cell wall of Gram-negative bacteria, it is reasonable to assume that blood endotoxin is translocated from the gut. In the present study, the fact that all patients with COVID-19-related pneumonia had negative blood cultures simultaneously with endotoxemia detection also points towards the gut origin of systemic endotoxemia.

Previous clinical studies have provided evidence for systemic endotoxemia in patients with severe COVID-19related pneumonia admitted to intensive care units (7). In the present study, we found no difference in systemic endotoxin levels between patients with severe and non-severe COVID19-related pneumonia, which indicates that gut-barrier integrity is affected early in the disease course. Endotoxin levels on admission were correlated with the inflammatory and prognostic markers CRP and ferritin. However, endotoxin levels on admission were not found to have any prognostic role, according to the presented results, since there was no difference between patients who progressed or not to SRF. A potential explanation of this finding might be that progression to SRF, which is usually associated with the development of an hyperinflammatory state, is not only dependent on the magnitude of inflammatory stimuli (e.g. endotoxin) but principally on the host inflammatory response (12). It has been shown that there is a complex interaction between the $\mathrm{S}$ protein of SARS-CoV-2 and endotoxin, which can lead to activation 

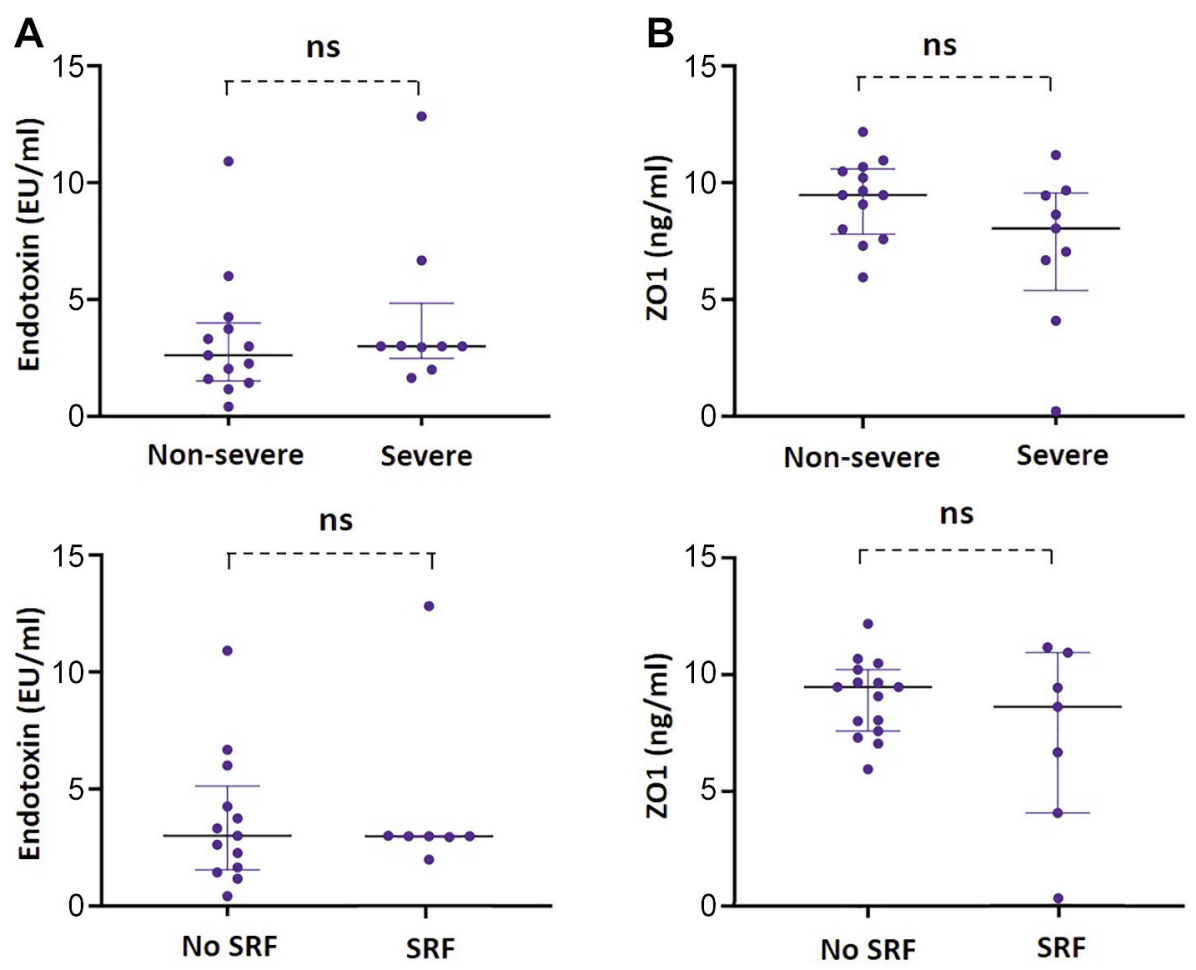

Figure 2. Comparison of serum levels of endotoxin $(A)$ and ZO1 (B) between patients with non-severe and severe COVID-19-related pneumonia on admission (upper panels) and between patients with and without progression to severe respiratory failure (SRF) requiring mechanical ventilation (lower panels). Lines and bars represent median and interquartile range, respectively. ns: Non-significant.

of pro-inflammatory nuclear factor kappa B in monocytes, thus promoting the subsequent cytokine response (13).

The mechanism of intestinal barrier dysfunction in COVID19-related pneumonia leading to systemic translocation of endotoxin might be multifactorial involving biological (gut microbiota), mechanical (intestinal epithelial cells and TJs) and immunological barriers (14). It has been demonstrated that patients with COVID-19-related gut-barrier dysfunction present significant alterations of their gut microbiota (6). To the best of our knowledge, this is the first study showing that the integrity of the intestinal paracellular barrier in patients with COVID-19-related pneumonia is impaired, as evidenced by their significantly higher serum ZO1 levels compared to healthy controls. The gut microbiota and the intestinal epithelium interact in a continuous so-called cross-talk because gut bacteria synthesize short-chain fatty acids such as butyrate, propionate and acetate, which are rich sources of energy for intestinal epithelial cells, while production of pathogen-associated molecular patterns contribute to gut immunomodulation, which interacts with both the innate and adaptive immune systems of the gut (15). Therefore, alterations of the gut microbiota in COVID-19 might represent a contributing factor implicated in impaired enterocyte TJ integrity, either through reduced energy supply to enterocytes or through the injurious effects of locally and systemically

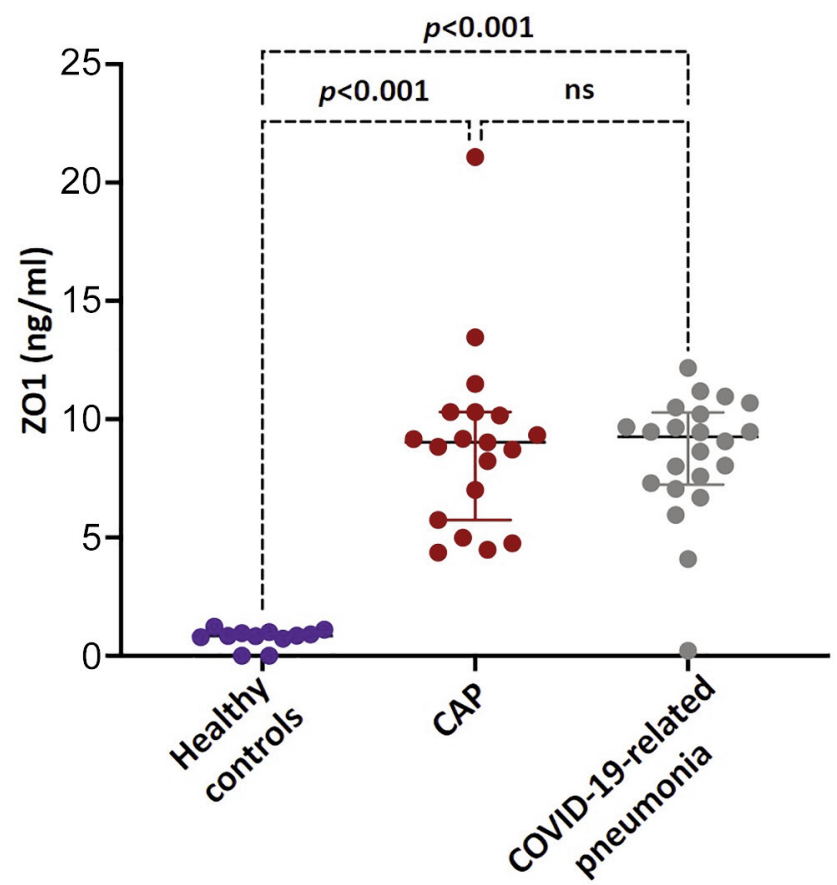

Figure 3. Serum zonula occludens-1 (ZO-1) levels in healthy controls, patients with non-COVID-19-related community-acquired pneumonia $(C A P)$ and those with COVID-19-related pneumonia. Lines and bars represent median and interquartile range, respectively. ns: Non-significant. 

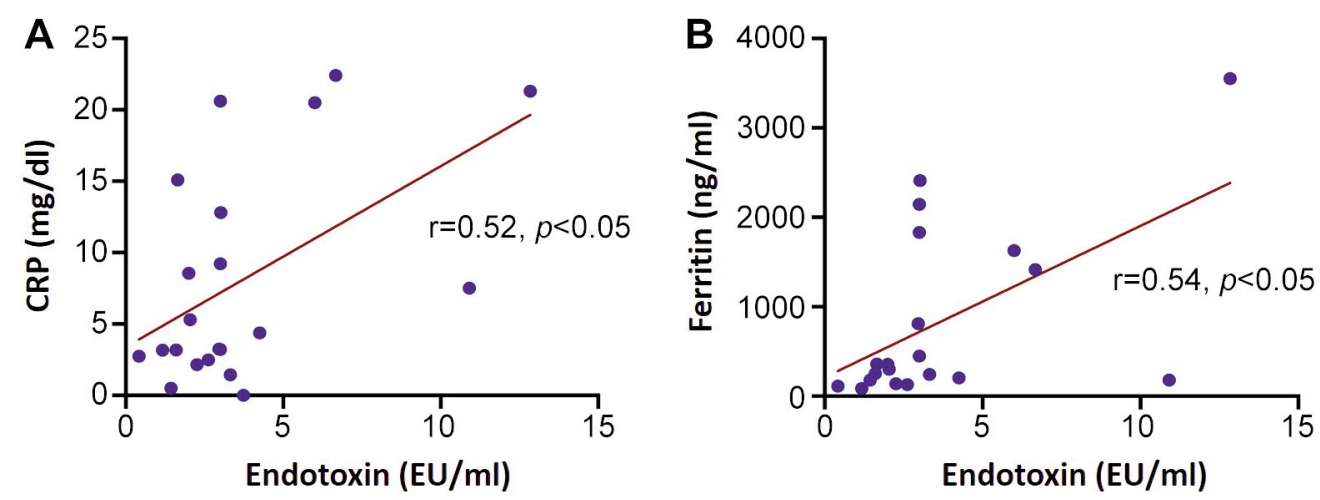

Figure 4. Correlations of serum leveIs of endotoxin with C-reactive protein $(C R P)(A)$ and ferritin $(B)$ in patients with COVID-19-related pneumonia.

produced proinflammatory cytokines (16). ZO1 is a $210-225$ $\mathrm{kDa}$ phosphoprotein that interacts with the TJ proteins occludin, claudins and junctional adhesion molecule, as well as $\mathrm{ZO} 2, \mathrm{ZO} 3$, afadin- 6 , cingulin and the actin cytoskeleton (16). Therefore, it plays a key role in bringing several components responsible for the paracellular barrier together and in connecting TJ proteins to the cytoskeleton (17). ZO1 was shown to be increased in serum in several gastrointestinal and non-gastrointestinal diseases associated with increased intestinal paracellular permeability due to TJ dysfunction, such as in coeliac disease, inflammatory bowel disease, obesity, diabetes type 1, rheumatoid arthritis and sepsis (18-21). According to our findings, serum ZO1 cannot be used to distinguish patients with COVID-19-related pneumonia according to their disease severity, nor does it appear to have a prognostic role for progression to SRF. Dysfunction of the paracellular function of the gut is an important determinant of gut-barrier failure and endotoxin translocation, but several other co-factors come into play when considering the potential development of the hyperinflammatory response termed a 'cytokine storm', which is associated with adverse outcomes $(3,22)$. In a recent small clinical study, we showed that increased circulating pro-coagulant phospholipids in patients with COVID-19-related pneumonia had a prognostic role for progression of patients to SRF (23).

Some limitations of the current study should be acknowledged. Firstly, a limited number of patients from one center were studied, which is a drawback especially when investigating the potential prognostic role of intestinal barrier biomarkers. Secondly, investigated biomarkers of gut-barrier function were evaluated only at one time point (on admission); therefore, this study does not provide information on their longitudinal dynamics, which have important pathophysiological value, especially for patients who consequently developed SRF. Thirdly, we did not directly assess the potential derangement of intestinal integrity by obtaining appropriate intestinal biopsy specimens with upper gastrointestinal endoscopy but instead chose to evaluate a non-invasive biomarker of barrier integrity.

In conclusion, the present study provides evidence that patients with COVID-19-related pneumonia present intestinal barrier dysfunction leading to systemic endotoxemia. Evaluated biomarkers of gut-barrier dysfunction and bacterial translocation on admission appear to have no prognostic role for progression to SRF. However, the role of gut-barrier dysfunction and bacterial translocation should not be neglected as an important component of the pathophysiology in such patients. The results of this pilot study should be further examined in larger prospective studies, potentially also examining the value of therapeutic measures aiming at preservation of gut-barrier integrity.

\section{Conflicts of Interest}

The Authors have no conflicts of interest to declare in relation to this study.

\section{Authors' Contributions}

SFA designed the study; DP, TC, CC and IO collected the samples; MM supervised clinical handling of patients; A-LdL performed the sample analyses; AM supervised laboratory analyses; DA and SM performed the statistical analysis and prepared the output of results; SFA wrote the article; and CC, MM and AM critically revised the article. All Authors have accepted responsibility for the entire content of this article and approved its submission.

\section{References}

1 Xie J, Tong Z, Guan X, Du B and Qiu H: Clinical characteristics of patients who died of Coronavirus disease 2019 in China. JAMA Netw Open 3(4): e205619, 2020. PMID: 32275319. DOI: 10.1001/jamanetworkopen.2020.5619

2 Liu D, Wang Q, Zhang H, Cui L, Shen F, Chen Y, Sun J, Gan L, Sun J, Wang J, Zhang J, Cai Q, Deng J, Jiang J and Zeng L: Viral sepsis is a complication in patients with Novel Corona 
Virus Disease (COVID-19). Med Drug Discov 8: 100057, 2020 PMID: 32838292. DOI: 10.1016/j.medidd.2020.100057

3 Assimakopoulos SF, Triantos C, Thomopoulos K, Fligou F, Maroulis I, Marangos M and Gogos CA: Gut-origin sepsis in the critically ill patient: pathophysiology and treatment. Infection 46(6): 751-760, 2018. PMID: 30003491. DOI: 10.1007/s15010-018-1178-5

4 Syed A, Khan A, Gosai F, Asif A and Dhillon S: Gastrointestinal pathophysiology of SARS-CoV2 - a literature review. J Community Hosp Intern Med Perspect 10(6): 523-528, 2020. PMID: 33194122. DOI: 10.1080/20009666.2020.1811556

5 Zhang Y, Cen M, Hu M, Du L, Hu W, Kim JJ and Dai N: Prevalence and persistent shedding of fecal SARS-CoV-2 RNA in patients with COVID-19 infection: A systematic review and meta-analysis. Clin Transl Gastroenterol 12(4): e00343, 2021. PMID: 33835096. DOI: 10.14309/ctg.0000000000000343

6 Gu S, Chen Y, Wu Z, Chen Y, Gao H, Lv L, Guo F, Zhang X, Luo R, Huang C, Lu H, Zheng B, Zhang J, Yan R, Zhang H, Jiang H, Xu Q, Guo J, Gong Y, Tang L and Li L: Alterations of the gut microbiota in patients with Coronavirus disease 2019 or H1N1 influenza. Clin Infect Dis 71(10): 2669-2678, 2020. PMID: 32497191. DOI: 10.1093/cid/ciaa709

7 Sirivongrangson P, Kulvichit W, Payungporn S, Pisitkun T, Chindamporn A, Peerapornratana S, Pisitkun P, Chitcharoen S, Sawaswong V, Worasilchai N, Kampunya S, Putcharoen O, Thawitsri T, Leelayuwatanakul N, Kongpolprom N, Phoophiboon V, Sriprasart T, Samransamruajkit R, Tungsanga S, Tiankanon K, Lumlertgul N, Leelahavanichkul A, Sriphojanart T, Tantawichien T, Thisyakorn U, Chirathaworn C, Praditpornsilpa K, Tungsanga K, Eiam-Ong S, Sitprija V, Kellum JA and Srisawat N: Endotoxemia and circulating bacteriome in severe COVID-19 patients. Intensive Care Med Exp 8(1): 72, 2020. PMID: 33284413. DOI: 10.1186/s40635-020-00362-8

8 COVID-19 Treatment Guidelines Panel. Coronavirus Disease 2019 (COVID-19) Treatment Guidelines. National Institutes of Health. Available at https://www.covid19treatmentguidelines. nih.gov/ [Last accessed on May 20 th, 2021 ]

9 Assimakopoulos SF, Tsamandas AC, Tsiaoussis GI, Karatza E, Triantos C, Vagianos CE, Spiliopoulou I, Kaltezioti V, Charonis A, Nikolopoulou VN, Scopa CD and Thomopoulos KC: Altered intestinal tight junctions' expression in patients with liver cirrhosis: a pathogenetic mechanism of intestinal hyperpermeability. Eur J Clin Invest 42(4): 439-446, 2012. PMID: 22023490. DOI: 10.1111/j.1365-2362.2011.02609.x

10 Metlay JP, Waterer GW, Long AC, Anzueto A, Brozek J, Crothers K, Cooley LA, Dean NC, Fine MJ, Flanders SA, Griffin MR, Metersky ML, Musher DM, Restrepo MI and Whitney CG: Diagnosis and treatment of adults with community-acquired pneumonia. An official clinical practice guideline of the American Thoracic Society and Infectious Diseases Society of America. Am J Respir Crit Care Med 200(7): e45-e67, 2019. PMID: 31573350. DOI: 10.1164/rccm.201908-1581ST

11 Kritselis I, Tzanetakou V, Adamis G, Anthopoulos G, Antoniadou E, Bristianou M, Kotanidou A, Lignos M, Polyzos K, Retsas T, Sassopoulou P, Papaioannou AI, Sinapidis D, Sereti K, Vittoros V, Ghanas P, Gogos C and Giamarellos-Bourboulis EJ: The level of endotoxemia in sepsis varies in relation to the underlying infection: Impact on final outcome. Immunol Lett 152(2): 167172, 2013. PMID: 23747516. DOI: 10.1016/j.imlet.2013.05.013

12 Torres Acosta MA and Singer BD: Pathogenesis of COVID-19induced ARDS: implications for an ageing population. Eur
Respir J 56(3): 2002049, 2020. PMID: 32747391. DOI: 10.1183/ 13993003.02049-2020

13 Petruk G, Puthia M, Petrlova J, Samsudin F, Strömdahl AC, Cerps S, Uller L, Kjellström S, Bond PJ and Schmidtchen AA: SARS-CoV-2 spike protein binds to bacterial lipopolysaccharide and boosts proinflammatory activity. J Mol Cell Biol 12(12): 916-932, 2021. PMID: 33295606. DOI: 10.1093/jmcb/mjaa067

14 Assimakopoulos SF, Triantos C, Maroulis I and Gogos C: The role of the gut barrier function in health and disease. Gastroenterology Res 11(4): 261-263, 2018. PMID: 30116424. DOI: $10.14740 / \mathrm{gr} 1053 \mathrm{w}$

15 Aggeletopoulou I, Konstantakis C, Assimakopoulos SF and Triantos C: The role of the gut microbiota in the treatment of inflammatory bowel diseases. Microb Pathog 137: 103774, 2019. PMID: 31586663. DOI: 10.1016/j.micpath.2019.103774

16 Assimakopoulos SF, Papageorgiou I and Charonis A: Enterocytes' tight junctions: From molecules to diseases. World J Gastrointest Pathophysiol 2(6): 123-137, 2011. PMID: 22184542. DOI: 10.4291/wjgp.v2.i6.123

17 Itoh M, Nagafuchi A, Yonemura S, Kitani-Yasuda T, Tsukita S and Tsukita S: The $220-\mathrm{kD}$ protein colocalizing with cadherins in non-epithelial cells is identical to ZO-1, a tight junctionassociated protein in epithelial cells: cDNA cloning and immunoelectron microscopy. J Cell Biol 121(3): 491-502, 1993. PMID: 8486731. DOI: 10.1083/jcb.121.3.491

18 Fasano A: Intestinal permeability and its regulation by zonulin: diagnostic and therapeutic implications. Clin Gastroenterol Hepatol 10(10): 1096-1100, 2012. PMID: 22902773. DOI: 10.1016/j.cgh.2012.08.012

19 Sapone A, de Magistris L, Pietzak M, Clemente MG, Tripathi A, Cucca F, Lampis R, Kryszak D, Cartenì M, Generoso M, Iafusco D, Prisco F, Laghi F, Riegler G, Carratu R, Counts D and Fasano A: Zonulin upregulation is associated with increased gut permeability in subjects with type 1 diabetes and their relatives. Diabetes 55(5): 1443-1449, 2006. PMID: 16644703. DOI: $10.2337 / \mathrm{db} 05-1593$

20 Wells JM, Brummer RJ, Derrien M, MacDonald TT, Troost F, Cani PD, Theodorou V, Dekker J, Méheust A, de Vos WM, Mercenier A, Nauta A and Garcia-Rodenas CL: Homeostasis of the gut barrier and potential biomarkers. Am J Physiol Gastrointest Liver Physiol 312(3): G171-G193, 2017. PMID: 27908847. DOI: 10.1152/ajpgi.00048.2015

21 Assimakopoulos SF, Akinosoglou K, de Lastic AL, Skintzi A, Mouzaki A and Gogos CA: The prognostic value of endotoxemia and intestinal barrier biomarker ZO-1 in bacteremic sepsis. Am J Med Sci 359(2): 100-107, 2020. PMID: 31812215. DOI: 10.1016/j.amjms.2019.10.006

22 Huang M, Cai S and Su J: The pathogenesis of sepsis and potential therapeutic targets. Int J Mol Sci 20(21): 5376, 2019. PMID: 31671729. DOI: 10.3390/ijms20215376

23 Assimakopoulos SF, Emmanuil A, Tsimeka A, Chalkidi T, Marangos $\mathrm{M}$ and Gogos C: Evidence for increased circulating procoagulant phospholipids in patients with COVID-19 pneumonia and their prognostic role. Clin Chem Lab Med 59(2): e53-e55, 2020. PMID: 33112778. DOI: 10.1515/cclm-2020-1260

Received May 6, 2021

Revised May 21, 2021

Accepted May 25, 2021 